Check for updates

Cite this: RSC Adv., 2018, 8, 26144

Received 24th May 2018

DOI: $10.1039 / c 8 r a 04451 a$

rsc.li/rsc-advances
Accepted 27th June 2018

\section{Green synthesis of safe zero valent iron nanoparticles by Myrtus communis leaf extract as an effective agent for reducing excessive iron in iron-overloaded mice, a thalassemia model}

\begin{abstract}
Shahram Eslami, ${ }^{a}$ Mohammad Ali Ebrahimzadeh (D) *a and Pourya Biparva*b
Green synthesis of Myrtus communis-Zero Valent Iron Nanoparticles (MC-ZVINs) was carried out in an alkaline environment. These nanoparticles were introduced for testing in vivo iron chelation in an ironoverload thalassemia model in mice. The MC-ZVINs were characterized by the use of imaging (electron microscopy) and spectroscopic (UV-vis and infrared absorption, dynamic light scattering and X-ray diffraction) methods. These methods demonstrated that some polyphenols are bound to the surfaces of the MC-ZVINs as a capping/stabilizing agent. The MC-ZVINs and defroxamine were injected 5 times a week over 4 weeks. The total iron and $\mathrm{Fe}^{3+}$ content of serum was determined by microwave plasmaatomic emission spectroscopy and an $\mathrm{Fe}^{3+}$ ion measuring kit respectively and liver enzyme levels were evaluated with an auto-analyzer. Morphological changes of the liver were examined by haematoxylin and eosin and Prussian blue staining. Additionally, the bioactivity of the iron nanoparticles was checked by $\mathrm{DPPH}$ and nitric oxide radical scavenging activity, reducing power and iron $\left(\mathrm{Fe}^{2+}\right)$ chelating ability. Iron-overloaded mice treated with the MC-ZVINs, indicated a drastic reduction in serum iron content when compared with the control groups and those treated with defroxamine. MC-ZVINs exhibit satisfactory potency to chelate excessive iron in mice. The data collected in this study will be helpful for selecting the most appropriate operational conditions for synthesizing green ZVINs in biomedical applications.
\end{abstract}

\section{Introduction}

There are multiple human disease conditions caused by the opportunistic deposition of toxic iron ions resulting in pathological illnesses, including death. $\mathrm{Fe}^{3+}$ intoxication is the focus of special attention since it may be amenable to treatment by in vivo chelation. For instance, major thalassemia, and other types of anemia are conditions treated by regular blood transfusions, which usually result in $\mathrm{Fe}^{3+}$ overload, following that generation of reactive oxygen species and degeneration of the liver, endocrine organs, myocardium, and brain. The preferred treatment for these conditions is applying iron chelators. Iron chelating treatment has been used for a long time as the standard care for patients who suffer from thalassemia and other kinds of anemia. Some iron chelators exist that are currently employed to manage and treat thalassemia and other transfusiondependent anemias including deferoxamine (DFO), deferiprone (L1) and deferasirox (ICL-670). In spite of the fact that

${ }^{a}$ Pharmaceutical Sciences Research Center, Hemoglobinopathy Institute, Department of Medicinal Chemistry, Faculty of Pharmacy, Mazandaran University of Medical Sciences, Sari, Iran. E-mail: zadeh20@gmail.com

${ }^{b}$ Department of Basic Sciences, Sari University of Agricultural Sciences and Natural Resources, Sari, Iran these drugs are highly effective, they have numerous side effects, making it necessary to replace them with safer alternatives. ${ }^{1}$

Recently, Zero-valent iron nanoparticles (ZVINs) have been proposed as a favorable technology and very effective choice for eliminating different metallic ions including $\mathrm{Pb}$ (II), $\mathrm{Cd}(\mathrm{II}), \mathrm{As}(\mathrm{V})$ and $\mathrm{Cr}(\mathrm{VI})$ in aqueous solutions. ${ }^{2}$ ZVINs have been widely applied due to their cost-effective and relatively environmentally friendly nature (lower toxicity). ${ }^{3}$ ZVINs, as a promising technology, are being applied for the successful treatment of various metallic ions in aqueous solutions. ${ }^{2}$ In the aforesaid applications, the intrinsic instability related to nanoparticles in the optimum size range over longer periods of time causes difficulty. To decrease the energy resulting from the high surface to volume ratio of the nanosized particles, they tend to form agglomerates. Moreover, bare metal nanoparticles are highly chemically reactive and can be easily oxidized in air which, on the whole, results in a loss of magnetism and dispersibility. Thus, from an applications point of view, it is essential to formulate protection strategies to stabilize naked magnetic nanoparticles against degradation during or post synthesis. ${ }^{4,5}$ There is still a great interest in using natural and biodegradable polymers (polyphenols) to cap these 
nanoparticles to prevent them from aggregation and oxidation. In this way, the application of biodegradable polymers as a protecting agent for ZVINs significantly enhances their applicability. Polyphenols have been selected as a reducing agent and stabilizing agent since polyphenols are biodegradable (nontoxic) and water soluble at room temperature, unlike other polymers. Second, polyphenols can form complexes with metal ions and which can subsequently reduce the metals. Third, due to the presence of alcohol functional groups, polyphenols can be used for reduction as well as stabilization of the nanoparticles. ${ }^{6}$ The field of magnetic zero-valent iron polymer nanoparticles is a newly-emerging area. The levels of magnetization of the carboxyl-terminated magnetic polymer nanoparticles suggest a 10 -fold increase in the saturation magnetization when compared with oxide-based nanoparticle suspensions, which underlines their potential use in biomedical applications. ${ }^{7}$ Multiple methods have been employed for producing ZVINs including thermal and sonochemical decomposition of iron-containing complexes, vacuum sputtering and reduction of $\mathrm{Fe}^{2+}$ or $\mathrm{Fe}^{3+}$ to ZVINs with sodium borohydride which is the most prevalent and traditional chemical method of reduction. ${ }^{8}$ Nevertheless, these methods have some limitations and serious problems, such as the application of hazardous reducing agents or precursors and agglomeration of nanoparticles that leads to the formation of larger aggregates because of van der Waals and magnetic forces. ${ }^{6}$ In addition, these procedures generate hazardous by-products that could directly influence the environment. ${ }^{9}$ As a result, the use of plants for synthesizing nanoparticles could be useful since plants are biorenewable resources and can possibly offer sustainable solutions for large-scale deployment. ${ }^{\mathbf{1 0}}$ So far, few techniques have been proposed for the preparation of ZVINs. The nanoparticles that are prepared by these methods suffer from relatively short life-times. The crucial aspect of their extreme reactivity is investigated in this research as the relative stability of such ZVINs has been increased using green tea polyphenols which cap the ensuing nanoparticles. ${ }^{\mathbf{1 1}}$ In this paper, we examine the biosynthesis of iron nanoparticles through the reduction of iron ions using methanolic Myrtus communis leaf extract (MCLE). Experimental and clinical studies demonstrate that $M$. communis has a wider spectrum of pharmacological and therapeutic effects such as antioxidative, antidiabetic, anticancer, antiviral, antibacterial, antifungal, neuroprotective and hepatoprotective activity. ${ }^{12}$ In addition to its low toxicity and biodegradability, $M$. communis is a medicinal herb applied in traditional medicine throughout the world. ${ }^{13}$ In research based on FTIR reports and a literature review, ${ }^{\mathbf{1 2}}$ it is observed that hydrolyzable tannins (plant polyphenols) were the major metabolites (79\%) existing in the leaf extract compared with other phytochemicals. Hydrolyzable tannins are a typical kind of polyphenols, mixed gallotannin/ellagitannins consisting of gallic acid/ellagic acid esters of glucose. ${ }^{14}$ Tannins displayed more powerful antioxidant activity relative to phenolic acids and flavonoids. ${ }^{15}$ It is stated that tannic acid (TA), which is a hydrolyzable form of tannins, can inhibit hydroxyl radical formation from the Fenton reaction through complexing ferrous ions. ${ }^{\mathbf{1 6}}$ Extracts which are rich in tannins might be able to prevent iron-mediated Fenton reaction toxicity. The application of $M$. communis is of interest not only for synthesizing iron nanoparticles but also for preventing the disorders associated with the resulting nanoparticle-mediated oxidative stress. In terms of synthesis, redox processes in the reaction are dependent upon the standard reduction potentials $\left(E^{0}\right)$ of the reagents. While the reduction potential values for plant polyphenols $\left(E^{0}\left[\mathrm{Ar}-\mathrm{OH}(\mathrm{I}) / \mathrm{Ar}-\mathrm{O}^{-}\right]\right.$, where $\mathrm{Ar}$ stands for phenyl group) range from 0.3 to $0.8 \mathrm{~V},{ }^{6,17}$ the reduction potential of Fe metal is strongly negative $(-0.44 \mathrm{~V})$. Hence, plant polyphenols themselves are not appropriate for the reduction of $\mathrm{Fe}^{3+}$ and $\mathrm{Fe}^{2+}$ ions to zero-valent iron and only polyphenol-assisted reduction of $\mathrm{Fe}^{3+}$ to $\mathrm{Fe}^{2+}$ is possible (the reduction potential of $\mathrm{Fe}^{3+} / \mathrm{Fe}^{2+}$ is $0.771 \mathrm{~V}$ ) as well as polyphenol-assisted reduction of silver $\left(E^{0}\left[\mathrm{Ag}^{+} / \mathrm{Ag}\right]=0.799 \mathrm{~V}\right)$ and other ions to their corresponding nanoparticles. According to recent investigations, iron is not reduced to ZVINs by polyphenols but probably forms an iron(III)-polyphenol complex (determined by energy dispersive spectrophotometry). ${ }^{\mathbf{1 0}}$ Compared to plant polyphenols, the standard redox potentials for the plant polyphenol anions follow a different trend. The standard redox potentials of various types of organic anions $\left(E^{0}\left[\mathrm{Ar}-\mathrm{O}^{-} / \mathrm{Ar}-\mathrm{O}^{2-}\right]\right)$ are approximately -0.88 to $-1.81 \mathrm{~V}$, more negative than the standard redox potential of Fe metal $(-0.44 \mathrm{~V}) .{ }^{18}$ Accordingly, it is concluded that polyphenol anion-assisted reduction of $\mathrm{Fe}^{3+}$ and $\mathrm{Fe}^{2+}$ ions to zero-valent iron $\left(\mathrm{Fe}^{0}\right)$ is possible. So, in this study, we have synthesized MC-ZVINs in an alkaline environment, and afterwards assessed the antioxidant characteristics of the resulting nanoparticles. In the alkaline environment, the hydroxyl and carboxylic groups of polyphenolic compounds contained in the MCLE are deprotonated and are made stronger as a complexing and reducing agent for the iron ions. Subsequently, the iron ions oxidized the hydroxyl groups into carbonyl groups in the reduction reaction as the iron ions were concurrently reduced into the ZVINs. Multiple investigations have recently indicated that an alkaline medium is a precondition for synthesizing metallic nanoparticles using active reducing components from bio-resources. ${ }^{\mathbf{1 9 2 0}}$

Currently, there is a growing interest in the application of natural products, which are safer, instead of chemical ones. In accordance with this approach, the study of green synthesized ZVINs is of special interest as they usually contain great amounts of bioactive compounds. Owing to the high tannin content and good antioxidant activities of MC-ZVINs, these nanoparticles were nominated as an assay for in vivo chelation testing. To our knowledge, the present research is the first study conducted to investigate the ability of green synthesized ZVINs to chelate excessive iron in iron-overloaded mice.

\section{Results and discussion}

In general, nanoparticles are classified based on their shape, size and dispersity. In numerous applications, these attributes play a key role. ${ }^{30}$ The green synthesized ZVINs' formation was confirmed and characterized (using imaging and spectroscopic techniques) and the antioxidant characteristics of the resulting nanoparticles were subsequently assessed. Then, the iron 
chelating activity of the nanoparticles in iron overloaded mice was tested and the serum iron content, levels of serum liver enzymes and pathological changes in animal liver tissue were measured.

\section{UV-vis spectral analysis}

As provided in Fig. 1, MC-ZVIN formation was suggested by the change in the color of the solution to black ${ }^{8}$ following the addition of $\mathrm{MCLE}$ to a $\mathrm{FeCl}_{3}$ solution (at alkaline $\mathrm{pH}$ ) after 30 minutes of incubation. Different plants have been applied to synthesize iron nanoparticles, but only a few reports are available which explain that the black coloration formed as a result of the oscillation of conduction band electrons of $\mathrm{Fe}^{0}$, known as the surface plasmon resonance (SPR). The MC-ZVINs formation was further confirmed by spectrophotometric absorption measurements in the wavelength of about $260 \mathrm{~nm}$ which is the signature band for $\mathrm{ZVINs}^{9}$ (Fig. 1). In order to confirm that the SPR band occurred because of ZVIN formation, spectra of the leaf extract and $\mathrm{FeCl}_{3}$ solution were recorded and positive control ZVINs which had been synthesized using sodium borohydrate (SB-ZVIN) were provided. ${ }^{2}$ MCLE strongly absorbs in the UV-vis region at about 210, 260 and $340 \mathrm{~nm}$ in aqueous media. Nonetheless, the $\mathrm{FeCl}_{3}$ and MCLE mixture during the preparation of the ZVINs showed a prominent peak at about $260 \mathrm{~nm}$ along with another sharp peak at around $210 \mathrm{~nm}$ which shows an association of MCLE with $\mathrm{Fe}^{0}$. These peaks indicate a strong binding-like Metal-Ligand interaction between $\mathrm{Fe}^{0}$ and the polyphenolic compounds existing in MCLE via the $\mathrm{C}=\mathrm{O}$ group. ${ }^{31,32}$ In this regard, the single fine band at $260 \mathrm{~nm}$ suggests the formation of ZVINs and the presence of a peak at $210 \mathrm{~nm}$ corresponds to the biomolecule capping on to the ZVINs' surface.

\section{FTIR spectral analysis}

The FTIR spectrum of the leaf extract indicates intense bands at $3420.71 \mathrm{~cm}^{-1}$ (O-H stretching vibrations), $2929.07 \mathrm{~cm}^{-1}(\mathrm{C}-\mathrm{H}$

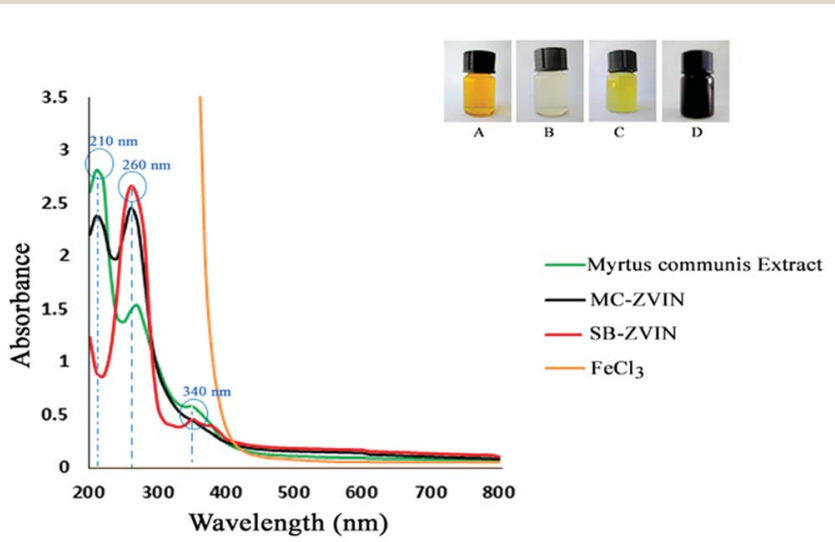

Fig. $1 U V$-vis spectrum of a $12 \mathrm{~g} \mathrm{~L}^{-1}$ aqueous solution of $M$. communis leaf extract in the presence of $\left[\mathrm{Fe}^{3+}\right]=0.1 \mathrm{M}$ at room temperature, inset showing the change in color of the materials involved in synthesis of stabilized zero-valent iron nanoparticles, (A) iron(II) chloride, (B) iron(III) chloride after adding ascorbic acid, (C) M. communis leaf extract, (D) colloidal solution of the resultant zero valent iron nanoparticles. and $\mathrm{CH}_{2}$ vibration of aliphatic hydrocarbons), $1718.34 \mathrm{~cm}^{-1}$ $\left(\mathrm{C}=\mathrm{O}\right.$ stretching vibration), $1617.70 \mathrm{~cm}^{-1}(\mathrm{C}=\mathrm{C}$ stretching vibrations), $1452 \mathrm{~cm}^{-1}$ (O-H bending vibrations), $1350.81 \mathrm{~cm}^{-1}$ (C-O stretching of the ester group), $1222 \mathrm{~cm}^{-1}$ (C-O asymmetric stretching in cyclic polyphenolic compounds) and $1041.08 \mathrm{~cm}^{-1}$ (O-H deformation) (Fig. 2a). The FTIR spectrum of the ZVINs displays intense bands at $3454.61 \mathrm{~cm}^{-1}(\mathrm{O}-\mathrm{H}$ stretching vibrations), $2926.27 \mathrm{~cm}^{-1}\left(\mathrm{C}-\mathrm{H}\right.$ and $\mathrm{CH}_{2}$ vibration of aliphatic hydrocarbons), $1719.06 \mathrm{~cm}^{-1}\left(\mathrm{C}=\mathrm{O}\right.$ stretching), $1627.23 \mathrm{~cm}^{-1}$ ( $\mathrm{C}=\mathrm{C}$ stretching vibrations), $1384.21 \mathrm{~cm}^{-1}$ (C-O stretching of the ester group) and $1073.89 \mathrm{~cm}^{-1}$ (O-H deformation), as well as absorption bands at around 539.45 and $459.07 \mathrm{~cm}^{-1}$ which refer to Fe-O stretches of iron oxide ${ }^{17,33}$ (Fig. 2b). Comparison of the IR spectrum of MCLE and the MC-ZVINs exhibits a band shift towards higher frequencies from 3420.71 to $3454.61 \mathrm{~cm}^{-1}$ (O-H stretching vibrations), 1350.81 to $1384.21 \mathrm{~cm}^{-1}$ (C-O stretching of the ester group) and 1718.34 to $1719.06 \mathrm{~cm}^{-1}(\mathrm{C}=$ O stretching) along with the presence of $1627.23 \mathrm{~cm}^{-1}(\mathrm{C}=\mathrm{C}$ stretching vibrations) suggesting the possible association of the polyphenolic compounds in the extract (mainly tannins) in the reduction process through the hydroxyl group and binding to the ZVINs through the carbonyl groups. It is also possibly due to the presence of biochemical molecules adjacent to the iron core, which has a paramagnetic effect.

\section{XRD}

The XRD pattern of the MC-ZVINs is provided in Fig. 3. The pattern is deficient in distinctive diffraction peaks and indicates that the synthesized MC-ZVINs are largely amorphous in nature. The broad shoulder peaks at $2 \theta=17^{\circ}$ and $32^{\circ}$ can be recognized as bio-organic compounds adsorbed from MCLE as a capping/ stabilizing agent, which was consistent with the FTIR results in Fig. 2. Furthermore, slight characteristic peaks appearing at about $2 \theta$ of $44-45^{\circ}$ corresponding to zero-valent iron $(\alpha-\mathrm{Fe})$ were also observed. ${ }^{8}$ A similar pattern for the ZVINs was identified when synthesized using Terminalia chebula aqueous extract. ${ }^{17}$

\section{Electron microscopy analysis}

The SEM and TEM images of the MC-ZVINs are presented in Fig. $4 \mathrm{~A}$ and $\mathrm{B}$, and demonstrate the successful synthesis of

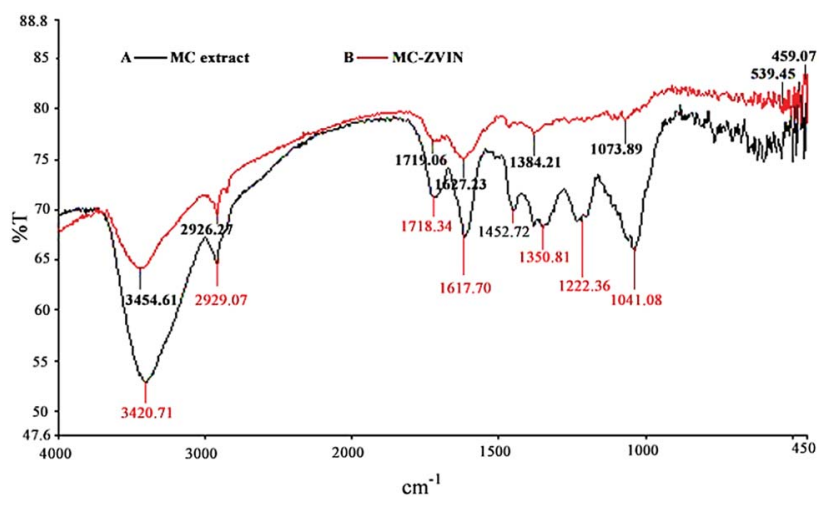

Fig. 2 FTIR spectrum of (A) the leaf extract; (B) the leaf extract stabilized zero valent iron nanoparticles. 


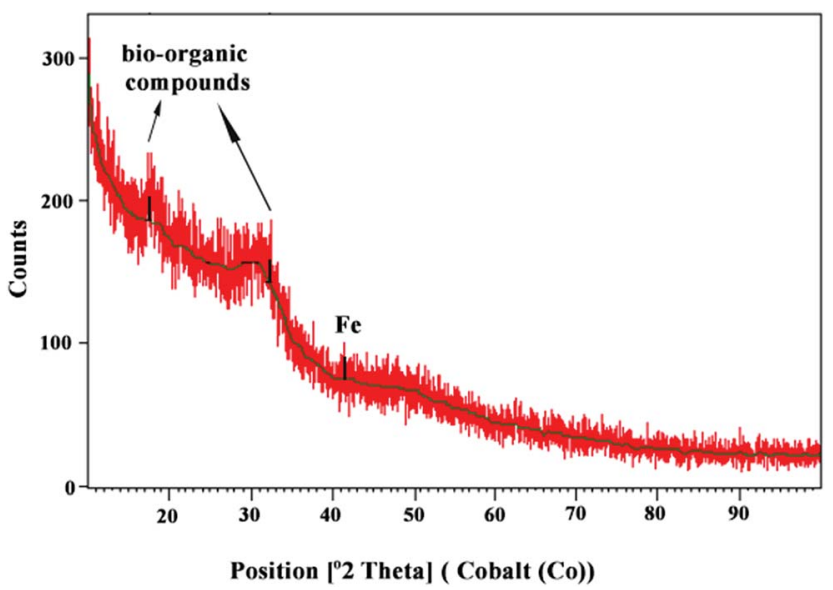

Fig. 3 XRD pattern of iron nanoparticles obtained using the leaf extract.

nanoparticles. It is obvious that the particles appear spherical with a diameter ranging between 40 and $60 \mathrm{~nm}$. The TEM image clearly exhibits a transparent organic layer coating around the ZVINs, which was due to the surrounding phytochemicals that serve as a capping agent to prevent agglomeration. Polyphenols or antioxidants in $M$. communis leaves probably play an

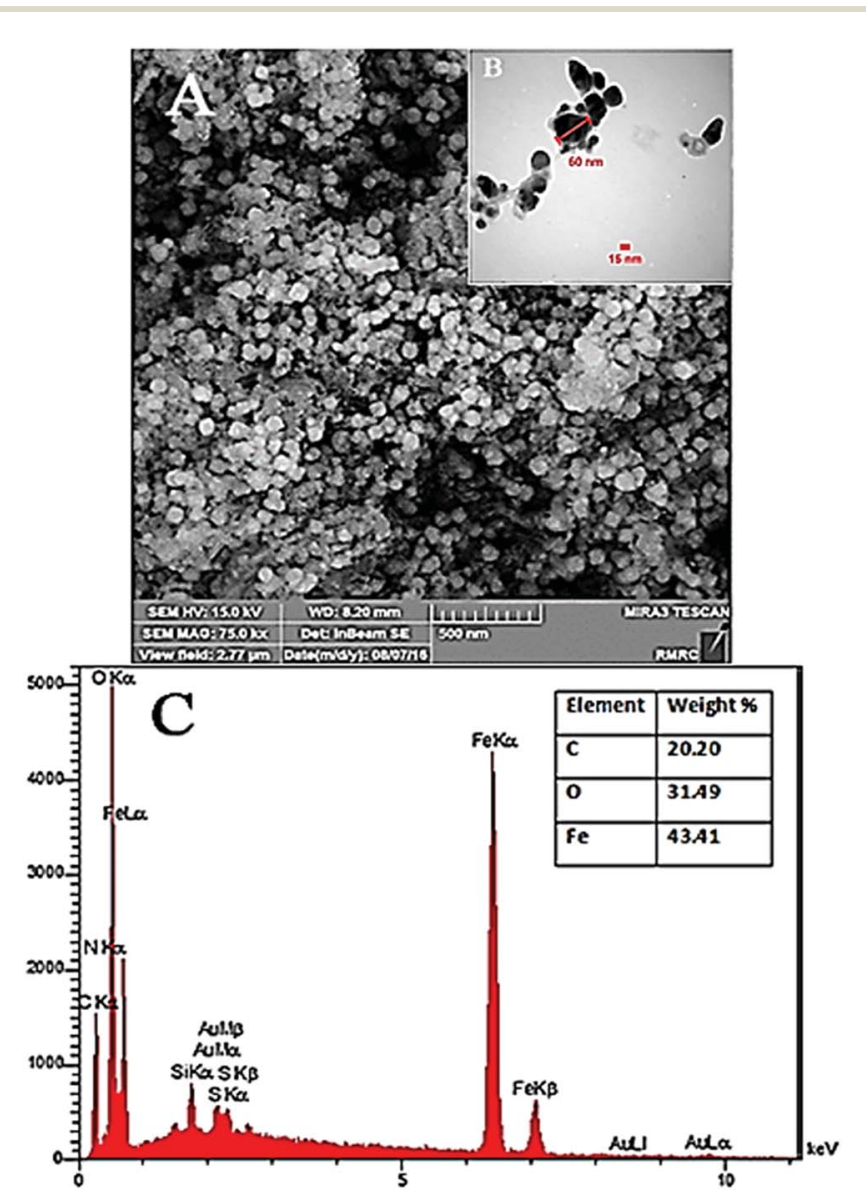

Fig. 4 (A) SEM image, (B) TEM image and (C) EDS results showing the presence of zero valent iron nanoparticles and the bio-organic components of M. communis leaf extract. important role in controlling the aggregation of the nanoparticles and do improve their dispersion by acting as a capping agent. The leaves with high antioxidant capacity and phenolic contents $^{12}$ could be a proper candidate for synthesizing different metal nanoparticles with uniform size. For better understanding of ZVIN synthesis, the localized elemental information of the MC-ZVINs was checked by EDS as displayed in Fig. 4C, which involves intense peaks of $\mathrm{C}, \mathrm{O}$ and $\mathrm{Fe}$, confirming the existence of $\mathrm{Fe}$. The $\mathrm{C}$ and $\mathrm{O}$ signals are attributed chiefly to the polyphenol groups and other $\mathrm{C}$ and O-containing molecules in MCLE. The weight composition of Fe in the MCZVINs synthesized by MCLE (43.41\%) is greater than the highest reported amount (42.98\%), which belongs to green tea extract. ${ }^{8}$ Perhaps, the reason is that $M$. communis has almost the greatest phenolic content among various leaves. ${ }^{12}$ However, unlike tea leaves which are commonly expensive, using $M$. communis leaves can add value to a non-usable waste product.

\section{Stability of the synthesized ZVIN}

The zeta potential is a criterion for measuring the magnitude of electrostatic repulsion or attraction between particles in a liquid suspension. It is one of the main parameters for characterizing the stability of nanoparticles in an aqueous environment. Particles with zeta potentials higher than $+30 \mathrm{mV}$ and lower than $-30 \mathrm{mV}$ are regarded as being stable for colloidal dispersion in the absence of steric stabilization. ${ }^{34}$ The bio-synthesized ZVINs' stability was assessed with a zeta potentiometer after synthesizing the metal nanoparticles (Fig. 5). The zeta potential of the MC-ZVINs $(-22.4 \mathrm{mV})$ revealed that stable dispersal in deionized (DI) water was more prolonged for the MC-ZVINs than the uncoated ZVINs $(-2.43 \mathrm{mV}) \cdot^{35}$ It is concluded that the capping bio-molecules existing on the surface of the ZVINs largely consist of negatively charged groups and are also responsible for greater stability of the extract-mediated ZVINs relative to the uncoated ZVINs. Significantly, the MC-ZVINs are strongly anionic ${ }^{36}$ and might be regarded as being quite nontoxic. ${ }^{37}$ This is based on the research conducted by Goodman et al. (2004) that demonstrated that the toxicity of metallic nanoparticles is associated with their interactions with

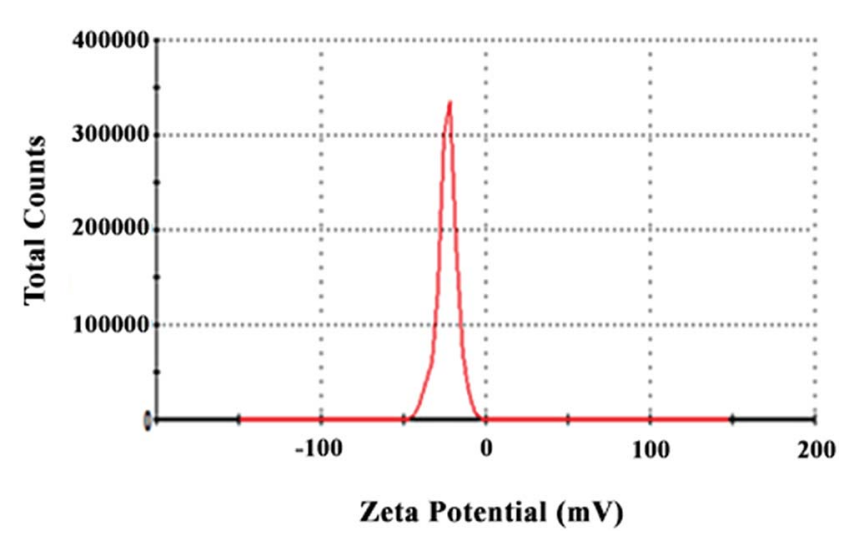

Fig. 5 Zeta potential of biosynthesized zero valent iron nanoparticles synthesized using the leaf extract. 
the cell membrane, a property initially mediated by their strong electrostatic attraction to the negatively charged layer. Their studies indicated that cationic particles are toxic, while particles with high negative zeta potential $\left(\mathrm{Zp}_{(\text {MC-ZVIN })}=-22.4 \mathrm{mV}\right)$ are quite nontoxic. $^{38}$

\section{Test for tannin compounds}

To prove the role of tannin compounds in the reduction process, a ferric ion reducing test was carried out. The addition of ferric chloride solution to the MCLE caused a color change to a dark blue green color. The fast color change to dark blue suggested the presence of tannins in the leaf extract (Fig. 6). According to this test, tannin compounds in the MCLE were responsible for the reduction of $\mathrm{Fe}(\mathrm{III})$ to $\mathrm{Fe}(0) .{ }^{21}$

\section{Content of total phenols, tannins, flavonoids, and proanthocyanidins (condensed tannins)}

Phenolic compounds such as phenolic acids, flavonoids, and tannins are widely distributed in plants, and have been the focus of great attention because of their antioxidant activities, which potentially have useful implications for the health of human beings. ${ }^{38}$ The phenolic content of the nanoparticles was evaluated to determine the total amount of phenolic compounds contained within the MC-ZVINs, and which showed the attachment of significant amounts $(211.28 \mathrm{mg}$ gallic acid equivalent per g MC-ZVIN) of phenolic compounds. It should be

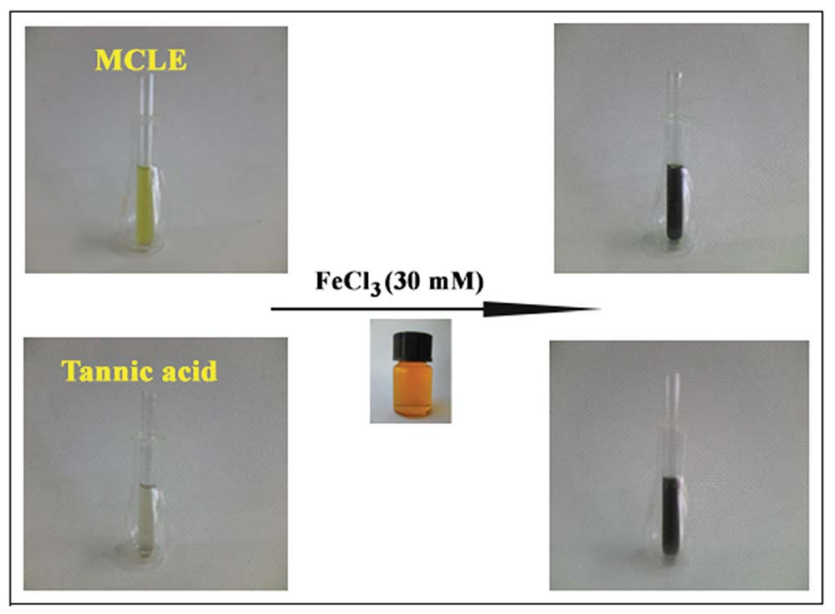

Fig. 6 Color change of MCLE and tannic acid by a ferric ion reducing test. noted that the literature review disclosed that the amount of total phenolic compounds in the biosynthesized iron nanoparticles has not been previously reported, which suggests that phenolic compounds existing in the extract are strongly attached to the metal core and cannot be easily separated from it in spite of washing with water and ethanol four times. Therefore, one might argue that this characteristic can be effective in decreasing the toxicity of the nanoparticles. Moreover, the total flavonoid content in the MC-ZVINs was expressed as a quercetin equivalent and it was found $50 \%$ relative to MCLE by reference to a standard curve $\left(y=0.0067 x+0.0132, r^{2}=\right.$ $0.999)$. It should be mentioned that the presence of flavonoids in the MC-ZVINs was $25 \%$ compared to phenolic contents. The hydrolyzable tannin content in the MC-ZVINs was expressed as a tannic acid equivalent and it was found 50\% relative to MCLE by reference to a standard curve $\left(y=0.0002 x+0.042, r^{2}=\right.$ 0.996). Because of the very low amounts of condensed tannins existing in the extract, roughly no amount of it was found in the MC-ZVINs. Table 1 briefly presents the results obtained from the quantitative determination of total phenols, flavonoids, tannins and proanthocyanidins (condensed tannins) from the MC-ZVINs and MCLE. It can be deduced from the results that MCLE was particularly rich in hydrolyzable tannins, of which the greatest combined value was found attached to the surface of the nanoparticles.

\section{In vitro antioxidant activity}

Plants include specific metabolites that are found to do a range of purposeful activities. It is stated in the literature that plant mediated nanoparticle synthesis requires sequential reduction followed by capping with these constituents of plants. ${ }^{39}$ Nevertheless, the interactions of these components with metals have not been properly investigated and the kind of activity they may confer to nanoparticles has not been reported until now. We are reporting, for the first time, the antioxidant activity of iron nanoparticles capped with plant constituents including free radical scavenging activity. The in vitro antioxidant activity of the MC-ZVINs and MCLE were measured in the present research and are provided in Table 2. According to the DPPH scavenging assay, the capped iron nanoparticles were recognized to be potent free radical scavengers when compared to the standards vitamin $\mathrm{C}$ and quercetin. The reducing power of a bioactive compound indicates the electron donating activity, which is an essential mechanism of phenolic antioxidant action and may function as a significant indicator of its potential

Table 1 Total phenolic, flavonoid, hydrolyzable tannin and condensed tannin contents of the MC-ZVINs and MCLE

\begin{tabular}{lcccc}
\hline Relative content $\left(\mathrm{mg} \mathrm{g}^{-1}\right.$ dry weight $)$ & & & \\
\hline Samples & Total phenols $^{a}$ & Flavonoids $^{b}$ & Hydrolyzable tannins $^{c}$ & \\
\hline MC-ZVINs & $211.28 \pm 6.34$ & $52.75 \pm 1.24$ & Condensed tannins $^{d}$ \\
MCLE & $346 \pm 10.86$ & $100.8 \pm 4.36$ & $139.53 \pm 5.29$ & $0.00 \pm 0.00$ \\
& & $277.89 \pm 7.32$ & $3.33 \pm 0.02$
\end{tabular}

${ }^{a}$ mg gallic acid equivalent per g sample. ${ }^{b} \mathrm{mg}$ quercetin equivalent per g sample. ${ }^{c} \mathrm{mg}$ tannic acid equivalent per g sample. ${ }^{d}$ mg leucocyanidin per $\mathrm{g}$ sample. ${ }^{e}$ All values (mean \pm standard deviation, $n=3$ ). 
Table 2 Antioxidant activities of the MC-ZVINs and MCLE ${ }^{b}$

\begin{tabular}{|c|c|c|c|c|}
\hline Sample & $\begin{array}{l}\text { DPPH free radical } \\
\text { scavenging IC50 }\left(\mu \mathrm{gl}^{-1}\right)\end{array}$ & $\begin{array}{l}\text { Nitric oxide scavenging } \\
\text { IC50 }\left(\mu \mathrm{g} \mathrm{ml} \mathrm{m}^{-1}\right)\end{array}$ & $\begin{array}{l}\mathrm{Fe}^{2+} \text { chelating } \\
\text { ability IC50 }\left(\mu \mathrm{g} \mathrm{ml}{ }^{-1}\right)\end{array}$ & Reducing power (EC50) ${ }^{a}$ \\
\hline MC-ZVINs & $11.42 \pm 0.96$ & $158.94 \pm 3.18$ & $629.65 \pm 4.74$ & $23.64 \pm 1.12$ \\
\hline Quercetin & $5.28 \pm 0.2$ & $20 \pm 0.1$ & - & - \\
\hline EDTA & - & - & $18 \pm 1.5$ & - \\
\hline Vitamin C & $5.05 \pm 0.1$ & - & - & $24.87 \pm 3.68$ \\
\hline
\end{tabular}

antioxidant activity. ${ }^{40}$ Astonishingly, MCLE displayed a 5-fold stronger reducing power activity relative to the standard antioxidant vitamin $\mathrm{C}\left(\mathrm{EC} 50_{\text {extract }}=1: 5\right.$ EC50 $\left.0_{\text {vitamin }} \mathrm{C}\right)$. These findings suggest the presence of specific bioactive compounds (high electron donors), which were responsible for producing the nanoparticles. Notably, the MC-ZVINs also showed comparatively better reducing power activity than standard vitamin C. Additionally, the MC-ZVINs demonstrated iron chelating activity around 5-fold better than MCLE. The high tannin content of MCLE and the MC-ZVINs appear to serve as good electron and hydrogen atom donors in the reduction process and continuing on, the MC-ZVINs should be able to quench DPPH and NO radicals. With respect to Table 2, the good iron chelating activity of the MC-ZVINs can simultaneously be associated with the good magnetic features of the metal core ${ }^{41}$ and the great reducing power of the biological molecules present in the extract attached to the surface of the nanoparticles. Consequently, the prepared nanoparticles can improve the medicinal characteristics of the plant in an innovative hybrid system (zero metal + biomolecule).

\section{Probable mechanism involved in the reduction and stabilization of ZVINs by the leaf extract}

ZVIN synthesis can hence be explained in terms of the polyphenols in the extract, the major ones being hydrolysable tannins. ${ }^{12}$ These tannins will undergo hydrolysis under alkaline conditions $(\mathrm{pH}=12)$ and turn to ellagic acid, gallic acid and glucose. $^{42}$ Additionally, gallic acid and ellagic acid in their anionic form are able to donate electrons (reducing power) compared to their non-anionic form (usual form) and to transform into their quinone form. This transformation of phenolics to their quinone forms allows for the reduction of an iron salt to zero valent iron. On the other hand glucose, the other hydrolyzed product of tannins, is a well known reductant, ${ }^{17}$ but glucose can only reduce $\mathrm{Fe}^{3+}$ to $\mathrm{Fe}^{2+}$, the resulting $\mathrm{Fe}^{2+}$ is then converted to $\mathrm{Fe}^{0}$ by the anionic forms of the other hydrolyzed products. The oxidized polyphenols function as good capping/stabilizing agent in the synthesis and dispersion of ZVINs which help in their sustained stability. The reduction and stabilization by the hydrolyzed products of tannins are also supported by the hard soft acid base (HSAB) principle. Based on the HSAB concept, the hydroxyl group $(-\mathrm{OH})$ is a hard ligand while the carbonyl group $(-\mathrm{C}=\mathrm{O})$ is a soft ligand in phenolic compounds. It has been previously indicated that hard ligands tend to reduce soft metals and soft ligands coordinate with the soft metals. ${ }^{43,44}$ Thus, based on the above-mentioned information, ZVIN formation can be explained as follows when the hard ligand $(-\mathrm{OH})$ existing in the hydrolyzed products of tannins comes in contact with the soft metal iron resulting in the reduction of the $\mathrm{Fe}^{3+}$ and $\mathrm{Fe}^{2+}$ ions to $\mathrm{Fe}^{0}$ and the hydrolyzed products of tannins undergo oxidation resulting in the formation of their respective quinine forms. The carbonyl group $(-\mathrm{C}=$ O) in the quinine form (IV, V, VI Fig. 7) is a soft ligand which coordinates with $\mathrm{Fe}^{0}$ through electrostatic interactions and helps to stabilize $\mathrm{Fe}^{0}$ through arresting its growth. A representative structural formula for one of the hydrolysable tannins in the extract (tellimagrandin II) has been provided in Fig. 7.

\section{Content of total iron in the plasma of iron overloaded and control mice}

The maximum amount of iron in the iron overloaded group obtained by MP-AES was equal to $3745.8 \pm 200.19 \mu \mathrm{g} \mathrm{dl}^{-1}$ which indicates a significant difference compared with the control

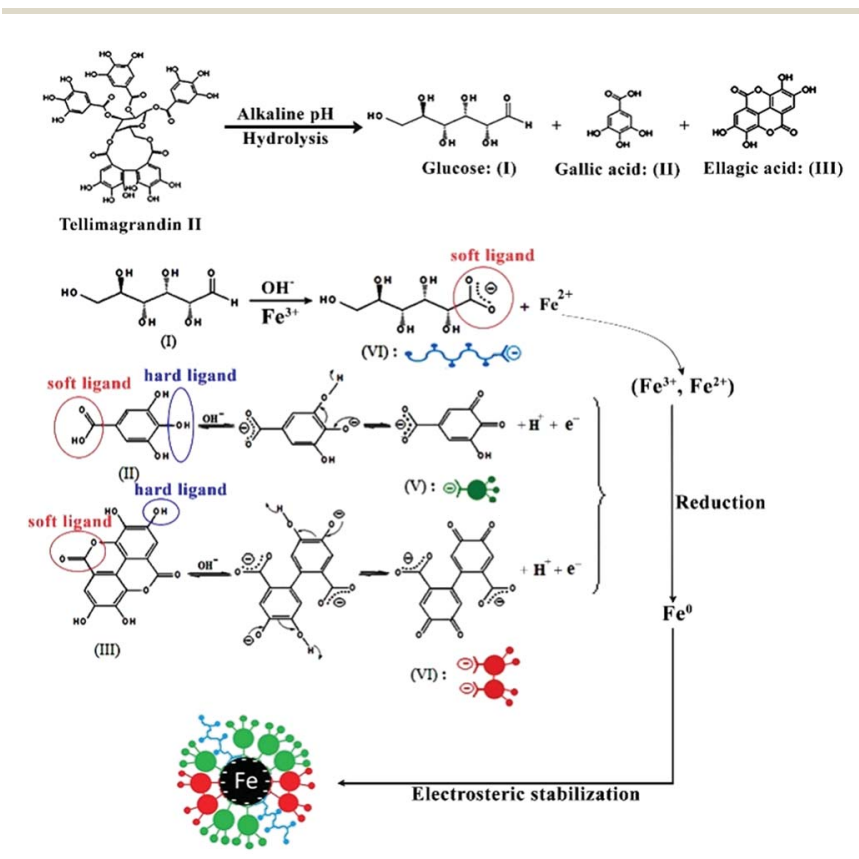

Fig. 7 Probable reaction mechanism of hydrolysable tannin-based reduction of iron salt. The phenolic groups in the tannins get oxidized to quinines with the subsequent release of electrons which reduce the iron ions. 
group at a statistical probability level of $p<0.001$. In the control group, the iron amount was $921 \pm 92.36 \mu \mathrm{g} \mathrm{dl}{ }^{-1}$. The iron overloaded mice treated with the MC-ZVINs revealed a reduction in their serum iron levels, among which those treated with $100 \mathrm{mg} \mathrm{kg}^{-1}$ of the MC-ZVINs exhibited the maximum reduction $\left(1207.2 \pm 63.79 \mu \mathrm{g} \mathrm{dl}^{-1}\right)$ (column E, Fig. 8a), reducing the iron content approximately to the levels that occur in the normal saline receiving group. The total iron serum level was observed to decrease in the mice treated with the MC-ZVINs, suggesting a significant difference $(p<0.001)$ compared to that of iron-overloaded mice (column B with D and E, Fig. 8a). Relative to the DFO-treated mice, the mice that received the $\mathrm{MC}$ ZVINs displayed lower levels of iron in their serum $(p<0.001)$, (column $\mathrm{C}$ with E, Fig. 8a). No difference exists between the iron-overloaded mice and the MCLE treated groups $(p>0.05)$, (column B with F and G, Fig. 8a). This shows that the reduction of excess iron over the extract is affected by the magnetic features of the nanoparticle metallic core. Furthermore, no difference exists between the group receiving only iron nanoparticles and the control group that is similar to the group that received only the extract. This demonstrates that nanoparticles and the extract in non-iron overloaded mice did not decrease
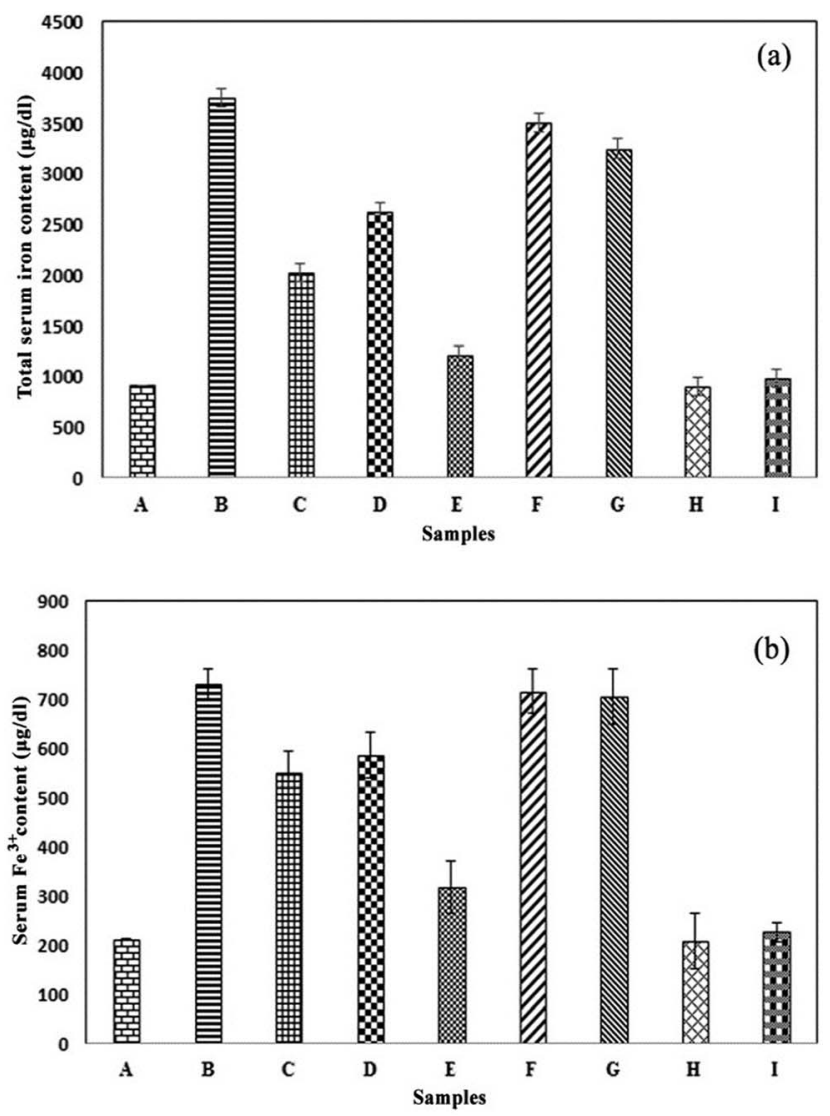

Fig. 8 (a) Total iron and (b) $\mathrm{Fe}^{3+}$ serum iron concentration determined by microwave plasma-atomic emission spectroscopy ((A) control group, (B) iron overloaded group, (C) DFO group, (D) iron overload + MC-ZVIN (50 $\mathrm{mg} \mathrm{kg}^{-1}$ ) group, (E) iron overload + MC-ZVIN (100 mg $\mathrm{kg}^{-1}$ ) group, (F) iron overload + MCLE $\left(50 \mathrm{mg} \mathrm{kg}^{-1}\right)$ group, (G) iron overload + MCLE (100 mg kg ${ }^{-1}$ ) group, (H) MC-ZVIN (100 $\mathrm{mg} \mathrm{kg}^{-1}$ ) and (I) MCLE (100 $\mathrm{mg} \mathrm{kg}^{-1}$ ) group) (means $\pm \mathrm{SD}, p<0.05$ ). the iron content and its value was close to that of the normal group.

\section{$\mathrm{Fe}^{3+}$ content in the serum of iron-overloaded mice}

The maximum amount of iron $\left(731.0 \pm 44.32 \mu \mathrm{g} \mathrm{dl}^{-1}\right)$ has been displayed in the iron-overloaded group by $\mathrm{Fe}^{3+}$ analysis using an iron kit which indicates a considerable difference compared with the control group $\left(213.6 \pm 29.77 \mu \mathrm{g} \mathrm{dl}^{-1}\right)$ at a statistical probability level of $p<0.001$, (column B with A, Fig. 8b). The iron overloaded mice treated with the MC-ZVINs exhibited an obvious reduction in $\mathrm{Fe}^{3+}$ content when compared with the control group. Once more, the MC-ZVINs displayed the greatest activity. The $\mathrm{Fe}^{3+}$ content of groups, receiving 50 and $100 \mathrm{mg}$ $\mathrm{kg}^{-1}$ of the MC-ZVINs were 585.2 \pm 54.12 and $318.53 \pm 44.60 \mu \mathrm{g}$ $\mathrm{dl}^{-1}$, respectively (Fig. 8b). As Fig. 8b shows, the MC-ZVINs reduce the serum iron to a level comparable to that of DFO (column E with C). Likewise, it has been demonstrated here that nanoparticles and extracts in iron non-overloaded mice did not decrease the $\mathrm{Fe}^{3+}$ content relative to the normal group.

\section{Toxicity study}

In this section, the potential inhibitory impacts of the MCZVINs in iron overloaded induced hepatotoxicity in mice were assessed by the levels of liver enzymes. Additionally, the protective effects of the nanoparticles in the liver of iron overloaded mice as shown by a reduction in tissue inflammation and iron deposition in histopathological examination relative to control animals is also discussed.

\section{Evaluation of serum AST, ALT and ALP enzymes}

AST, ALT and ALP enzymes can identify liver injury, especially AST, which is more specific to the livers of mice. Najafzadeh and colleagues argued that ALT and AST serum levels were directly involved in iron toxicity. ${ }^{45}$ Changes in liver function were evaluated by the levels of these enzymes (Fig. 9A-C), which were considerably increased in iron overloaded mice relative to the control group (AST and ALP; $P<0.05$, ALT; $P>0.05$ ). In comparison with the values in the iron overloaded mice, treatment with the MC-ZVINs remarkably reduced the levels of AST, ALT and ALP in a dose-dependent manner $(P<0.05)$. At the same time, AST, ALT and ALP levels of DFO-treated mice were also significantly reduced, compared with those of the iron overloaded mice (AST and ALP; $P<0.05$, ALT; $P>0.05$ ). Accordingly, treatment of iron overloaded mice with the MCZVINs greatly attenuated the hepatic dysfunction (ALT/AST/ ALP levels). In short, in the MC-ZVINs-treated group, the level of AST was lower than DFO-treated group and close to that the control group, but the levels of ALT and ALP were even lower than those of the control group.

\section{Histopathological examination of the liver by H\&E staining}

Light microscopy analysis of H\&E stained liver sections revealed that in the iron-overloaded group, the hepatocytes were necrotic and the hepatic lobule structure appeared disintegrated; on the other hand, proliferating fibrous tissues, pseudo lobules, bile 

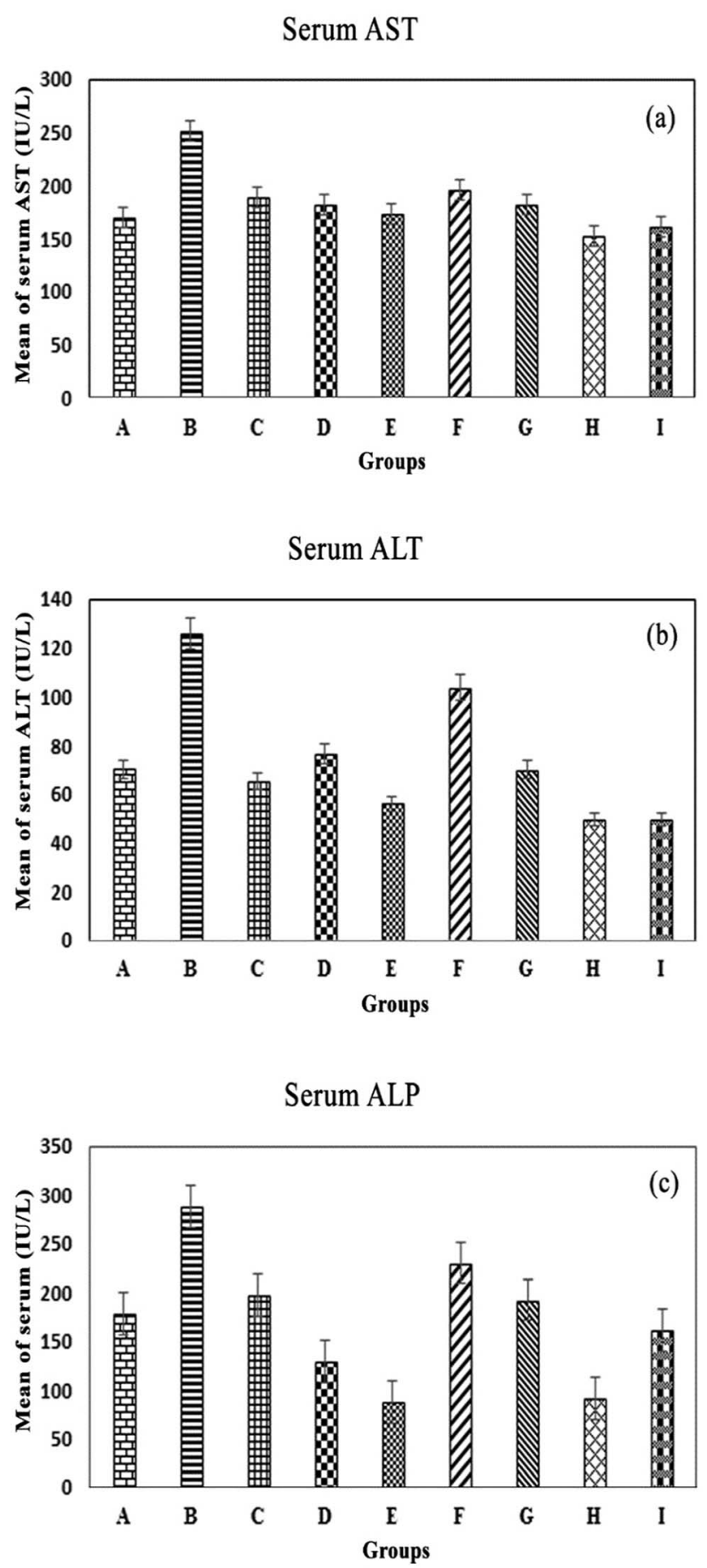

Fig. 9 Serum AST (a), ALT (b) and ALP (c) concentrations in mice ( $n=$ 6) ((A) normal saline, (B) iron dextran, (C) iron dextran + deferoxamine, (D) iron dextran + MC-ZVIN (50 $\left.\mathrm{mg} \mathrm{kg}^{-1}\right)$, (E) iron dextran + MC-ZVIN $\left(100 \mathrm{mg} \mathrm{kg}^{-1}\right)$, (F) iron dextran + MCLE (50 mg kg$\left.{ }^{-1}\right)$, (G) iron dextran + MCLE (100 $\left.\mathrm{mg} \mathrm{kg}^{-1}\right),(\mathrm{H}) \mathrm{MC}-\mathrm{ZVIN}\left(100 \mathrm{mg} \mathrm{kg}^{-1}\right)$ and (I) MCLE (100 mg $\left.\mathrm{kg}^{-1}\right)$ ) (means $\pm \mathrm{SD}, p<0.05$ ).

duct proliferation and extensive portal tract inflammation were observed, and haemorrhagic necrosis was discovered around the fibrotic tissues and dark brown particles (iron deposits) in the liver tissues. Additionally, obvious fatty degeneration of hepatocytes and scattered lymphocytes in filtration in the liver interstitium were detected (Fig. 10B). The H\&E staining result suggested that periportal inflammation, focal necrosis and genesis of brown pigments occurred in the mice receiving DFO, but to a lesser degree compared to that observed in the ironoverloaded mice (Fig. 10C).

The MC-ZVINs (100 mg per kg per day) treatment remarkably decreased the extent of necrotic hepatocytes, fibrous tissues and pseudo lobules, with little accompanying inflammatory cell infiltration (Fig. 10D). Meantime, severe portal inflammation with brown particles of hemosiderin was seen in iron overload mice treated with MCLE (Fig. 10E). Notably, in the group that received only the iron nanoparticles, slight portal inflammation was observed, whereas inflammation was not found in the group receiving the extract (MCLE) (Fig. 10F and G). Histopathological investigation found that treatment with the MCZVINs decreased iron deposition and ameliorated pathological changes in the livers of iron overloaded mice. The MC-ZVINs showed significant protective effects on the livers of iron overloaded mice, which were at least somewhat because of the decrease in iron deposition and the antioxidant characteristics of these nanoparticles.

\section{Histopathological examination of the liver by Prussian blue staining}

Among the organs of the body, the liver is more vulnerable to iron overloading damage; thus, we used liver tissue in a pathological investigation to demonstrate the iron overloading damage and to exhibit the potential inhibitory impacts of MCZVINs. Pathological changes in liver tissue were monitored by the Prussian blue staining method. Prussian blue to black stained iron deposits can be seen in the cytoplasm of hepatocytes in the iron-overloaded group (Fig. 11B). The iron content is reduced in liver tissue upon treatment with DFO and the MCZVINs. The MC-ZVINs are even more potent relative to DFO (Fig. 11C-E). On the other hand, only a minor change was detected in the amount of iron deposited in the control group (Fig. 11A). The excess iron deposition in hepatic parenchymal cells facilitates the production of free radicals - a significant pathway for hepatic injury. ${ }^{46}$ Since the MC-ZVINs have exhibited good iron chelating and free radical scavenging activity (Table

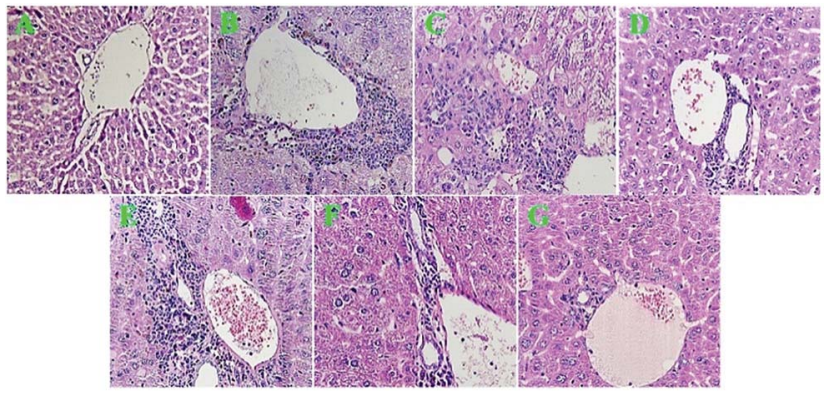

Fig. 10 Effects of the MC-ZVINs on iron deposition in mouse liver with H\&E. Representative microscopic photographs of livers stained with H\&E (magnification 400). ((A) control group, (B) iron overloaded group, (C) iron overload + DFO group, (D) iron overload + MC-ZVINs group, (E) iron overload + MCLE group, (F) MC-ZVINs group, (G) MCLE group). 


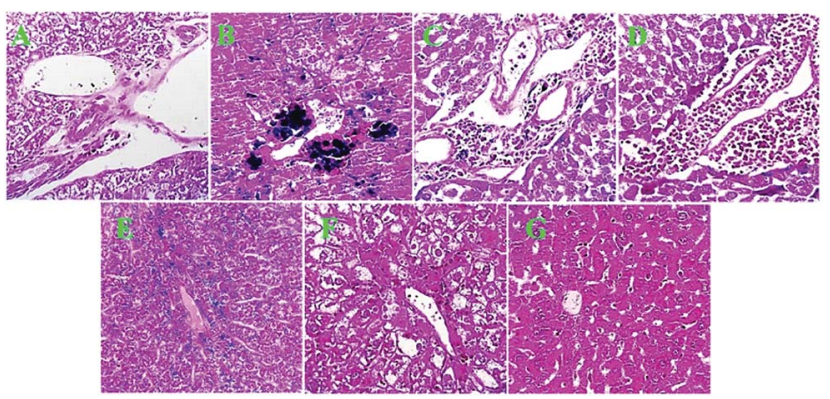

Fig. 11 Effects of MC-ZVINs on iron deposition in mouse liver Representative microscopic photographs of livers stained with Prussian blue (magnification 400). ((A) control group, (B) iron overloaded group, (C) iron overload + DFO group, (D) iron overload + MC-ZVINs group, (E) iron overload + MCLE group, (F) MC-ZVINs group, (G) MCLE group).

2), we hypothesized that they may be a favorable treatment for iron overload and liver damage induced in mice. Hence, in the present study, we examined the iron chelating activity of MCZVINs as a safe compound. Our results demonstrated that MC-ZVINs could reduce the level of iron in blood serum. Further, MC-ZVINs were also capable of diminishing the iron accumulation in liver tissue, leading to reduced tissue injury. The MC-ZVINs were also found to display useful features against iron accumulation in the liver tissue of mice. The hybrid nanoparticles were able to decrease tissue inflammation. Relative to DFO, the MC-ZVINs chelate more iron in liver tissues, as shown by Prussian blue staining (Fig. 11C-E). On the whole, MC-ZVINs were found to be an effective compound to prevent, or at least decrease, the deleterious impacts of excessive iron in mice. The protective influence of the MC-ZVINs (a nano-bio composite) in the livers of iron overload mice may be owing to both the antioxidant and iron chelation activities of the MCZVINs. Seemingly, this efficacy of MC-ZVINs can be assessed in clinical trials.

\section{Conclusions}

An innovative and promising green synthesis of iron nanoparticles using hydrolyzable tannins identified in $M$. communis is reported for the first time. The hydrolyzable tannins existing in the solvent extract serve both as reducing and capping/ stabilizing agents and thus are an alternative to chemical methods in which the applications of unsafe reagents and toxic expensive organic solvents are inevitable. The existence of the alkaline environment in the synthesis process raises the solubility of the methanolic extract in water, and converts the hydroxyl and carboxylic groups available in tannins to stronger reagents through de-protonation. Based on the TEM and SEM images, it is found that the average sized nanoparticles have a diameter of around $50 \mathrm{~nm}$. FTIR measurements provided powerful evidence that tannins form a coat covering the iron nanoparticles to stabilize and prevent their agglomeration. A colloidal solution of the MC-ZVINs shows good antioxidant characteristics and satisfactory potency to chelate excessive iron in mice, potentially presenting a new alternative approach to treat diseases correlated with iron overload. In comparison with defroxamine, the MC-ZVINs chelate more excess iron in serum and liver tissue, considerably attenuated the hepatic dysfunction (ALT/AST/ALP levels) and also decreased iron deposition and ameliorated pathological changes in the liver of iron overloaded mice as demonstrated by our findings, while its synthesis and use are cheap and convenient relative to defroxamine. It can be mentioned that this efficacy of the MC-ZVINs can be examined in clinical trials. In accordance with the results, the antioxidant activity, particularly the good iron chelating activity of the MC-ZVINs, can simultaneously be attributed to the good magnetism characteristics of the metallic core in the adsorption of iron ions and the electron-donating activity of the negatively charged functional groups $(\mathrm{Zp}=$ $-22.4 \mathrm{mV}$ ) present in the bio-molecules attached to the surface of the nanoparticles. All these results indicate a new pharmacological application of MC-ZVINs and present preliminary experimental support for their use as a therapeutic compound for iron overload related diseases.

\section{Materials and methods}

\section{Chemicals and reagents}

All chemicals used were analytical grade and were used without further purification. Iron(III) chloride $\left(\mathrm{FeCl}_{3}\right)$, Iron(II) chloride $\left(\mathrm{FeCl}_{2}\right)$, sodium hydroxide, sodium nitroprusside dehydrate, potassium hexacyanoferrate(III), tannic acid, ferrozine, $\mathrm{HCl}$, nitric acid, $n$-BuOH, sodium borohydrate, sodium carbonate, aluminum chloride, potassium acetate, vitamin $\mathrm{C}, \mathrm{Na}_{2}$ EDTA and Folin-Ciocalteau, were purchased from Merck (Darmstadt, Germany). Quercetin, gallic acid and DPPH were purchased from Sigma (St. Louis, USA). Deferoxamine was purchased from Novartis Pharma AG, Basel, Switzerland. Iron dextran was purchased from Vifor Pharma, UK. Distilled deionized water was prepared using an Ultrapure ${ }^{\mathrm{TM}}$ water purification system, Millipore SAS 67120 (Millipore Inc., Molsheim, France). $\mathrm{Fe}^{3+}$ ion measuring kit was obtained from ZiestChem Diagnostics (Tehran, Iran). Normal saline was obtained from the Shahid Ghazi Pharmaceutical Company (Tabriz, Iran).

\section{Plant material and leaf extract preparation}

Authenticated leaves of $M$. communis were gathered from the surrounding woods of Gachsaran, Lorestan, Iran. $50 \mathrm{~g}$ of the leaf powder was extracted at room temperature through the maceration method using $500 \mathrm{ml}$ of methanol. The obtained extract was filtered and concentrated in a rotary vacuum until a crude solid extract with a yield of $\sim 15 \%$ was achieved and kept at $4{ }^{\circ} \mathrm{C}$ until used for further work.

\section{Green bio-synthesis of zero valent iron nanoparticles}

The stabilized ZVINs were prepared by reducing $\mathrm{Fe}^{3+}$ to $\mathrm{Fe}^{0}$ using MCLE and ascorbic acid as an antioxidant agent to protect the iron(III) ions from oxidation without air evacuation. The synthesis process is based on the following: $12 \mathrm{~g} \mathrm{~L}^{-1}$ of the dried extract solution was prepared in $50 \mathrm{ml}$ of $0.1 \mathrm{M} \mathrm{NaOH}$ 
solution. $0.1 \mathrm{M}$ ascorbic acid and $0.1 \mathrm{M}$ iron chloride were prepared by dissolving solids in a $50 \mathrm{ml}$ ethanol/water mixture with a 30/70 ratio (v/v) and stirred well. The extract solution was poured in a burette and added drop by drop into the iron chloride solution at a volume ratio of $2: 1$ with strenuous shaking. The immediate appearance of a black color suggested the reduction of iron ions. The stirring was continued for another 30 minutes to complete the reaction. The achieved MCZVINs were centrifuged at $14000 \mathrm{rpm}(\mathrm{RCF}=20000)$ for 20 minutes, then washed three times using deionised water and ethanol to eliminate any unreacted biomolecules. The synthesized nanoparticles were redispersed in normal saline for in vivo studies. As a positive control, ZVINs synthesized using sodium borohydrate (SB-ZVIN) were also prepared for comparison.

\section{Apparatus}

UV-vis spectra were recorded as on a spectrophotometer (EZ201, Perkin Elmer, Wellesley, MA, USA). The size, morphology and composition of the biosynthesized ZVINs were characterized by transmission electron microscopy (EM 900, Zeiss, Oberkochen, Germany), scanning electron microscopy (MiraIII, Tescan Co., Brno, Czech Republic), X-ray diffraction spectroscopy (X'PertMPD, Philips Electronics, Almelo, Netherlands) and Fourier transform infrared spectroscopy (FTIR) (model Spectrum One, PerkinElmer, Wellesley, MA, USA). The stability of the developed ZVINs was analyzed using a Zetasizer Nano-series (model Zen 3600, Malvern, Worcestershire, UK). The iron content was determined by microwave plasma-atomic emission spectroscopy (model 4100, MP-AES Agilent Technologies, Inc., Santa Clara, CA, USA). The levels of liver enzymes in the serum samples were measured with an autoanalyzer (BT3000 PLUS, Biotechnica, Rome, Italy). The stained liver tissue sections were evaluated under a light microscope (Lx 400, Labomed, California, USA).

\section{Assay of tannin compounds}

A ferric ion reducing test was carried out to determine the existence of tannin compounds in MCLE. In short, a $\mathrm{FeCl}_{3}$ solution (30 mM) was added to MCLE to observe a color change. Condensed tannins gave a brownish green color and hydrolysable tannins displayed a blue-black color while creating ferrous compounds. ${ }^{21}$

\section{In vitro antioxidant assays}

The antioxidant activity of the MC-ZVINs (3.125-50 $\left.\mu \mathrm{g} \mathrm{ml}^{-1}\right)$ was assessed by DPPH assay based on a previously published method. ${ }^{22}$ The DPPH free radical scavenging activity of biosynthesized MC-ZVINs was stated as an inhibition percentage of DPPH calculated using the following formula. $\left[\left(A_{0}-A_{\mathrm{s}}\right) / A_{\mathrm{s}}\right] \times$ 100 , where $A_{0}$ was the absorbance of the control (DPPH radical + methanol) and $A_{\mathrm{s}}$ was the absorbance of sample/standard vitamin $\mathrm{C}$. The nitric oxide scavenging activity of the synthesized MC-ZVINs (6.25-800 $\mu \mathrm{g} \mathrm{ml}^{-1}$ ) was quantified through the reaction method provided by Sousa and colleagues. ${ }^{23}$ The results obtained were estimated using the above-mentioned formula and compared with the standard antioxidant quercetin. The chelation of ferrous ions by the synthesized MC-

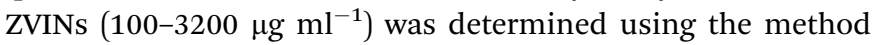
reported by Khalili and colleagues. ${ }^{24}$ The percentage inhibition of ferrozine- $\mathrm{Fe}^{2+}$ complex formation was estimated similar to above and $\mathrm{Na}_{2}$ EDTA was applied as the positive control. The total reducing power of the synthesized MC-ZVINs (25-400 $\mu \mathrm{g}$ $\mathrm{ml}^{-1}$ ) was evaluated by following the method reported by Ebrahimzadeh and colleagues. ${ }^{25}$

\section{Determination of total phenolic and total flavonoid content existing in the nanoparticles}

The total phenolic content was examined using the Folin-Ciocalteau colorimetric method. ${ }^{26}$ First, a nanoparticle sample was blended with Folin Ciocalteau's phenol reagent. Afterwards, a sodium carbonate solution was added to the obtained mixture and next, after vortex ( $5 \mathrm{~min}$ ), was incubated for 2 hours at room temperature. The absorbance of the reactions was assessed at $760 \mathrm{~nm}$ with a spectrophotometer. The total phenolic content was expressed as equivalents of gallic acid. The total flavonoid content was determined by employing the method developed by Chang et al. $^{27}$ In short, the NPs solutions were mixed with $10 \%$ aluminum chloride and $1 \mathrm{M}$ potassium acetate solution and stored at ambient temperature for 30 minutes. The absorbance of the reaction mixture was determined at $415 \mathrm{~nm}$. The total flavonoid content was calculated as quercetin from a calibration curve. All the experiments were carried out in triplicate.

\section{Determination of tannins}

The total hydrolyzable tannin content was examined using the potassium iodate colorimetric method. ${ }^{28}$ A calibration curve was prepared according to the standards of tannic acid. The concentration of MCLE was estimated by the use of this calibration curve and expressed as mg tannic acid equivalents $\mathrm{ml}^{-1}$. Condensed tannins in the extracts were determined based on the standard method of analysis of proanthocyanidins using an $n$-BuOH-HCI-Fe(III) mixture and based on the hydrolysis of proanthocyanidins to anthocyanidins. ${ }^{29}$ All the experiments were performed in triplicate.

\section{In vivo experiments}

Fifty four samples of male NMRI mice (20-25 g) were purchased from Pastor Institute (Amol, Iran) and kept inside polypropylene cages under laboratory conditions (temperature, $23 \pm$ $1{ }^{\circ} \mathrm{C}$, humidity $45-55 \%$, with a $12 \mathrm{~h}$ light: $12 \mathrm{~h}$ dark cycle (lights on at $7 \mathrm{am})$ ). The animals had free access to standard pellets and water. Experiments were performed between 8 am to $2 \mathrm{pm}$. Experiments were carried out following the approval of and based on the guidelines developed by the Animal Experimental Ethical Committee, The Mazandaran University of Medical Sciences (approval no. 911, 2012). The mice were assigned into nine groups as follows: (A) control (negative control), (B) ironoverloaded (positive control), (C) iron-overloaded + DFO, (D) iron-overloaded + MC-ZVINs (50 mg per kg per day), (E) ironoverloaded + MC-ZVINs (100 mg per kg per day), (F) iron overload + MCLE (50 $\mathrm{mg} \mathrm{kg}^{-1}$ ) and (G) iron-overloaded + MCLE (100 mg per kg per day) (H) MC-ZVINs (100 $\mathrm{mg} \mathrm{kg}^{-1}$ ) and (I) 
MCLE (100 $\mathrm{mg} \mathrm{kg}^{-1}$ ) groups. To induce iron overloading, the mice received $100 \mathrm{mg}$ per $\mathrm{kg}$ per day iron by intraperitoneal (i.p.) injections of iron dextran five days a week for four subsequent weeks and were then left for one month to equilibrate the excess iron. DFO was applied as a positive control group (1 mg per kg per day, i.p. injections, over a frequency of five days a week for four subsequent weeks and then left for 1 month). To assess the iron-chelation capacity of the MC-ZVINs, the colloidal solution of nanoparticles was administered as an i.p. injection five times a week at concentrations of 50 and $100 \mathrm{mg}$ per $\mathrm{kg}$ per day for MC-ZVIN over four weeks. Instead, the control ironoverloaded group received normal saline with the same frequency and duration. At the end of the experiment (after one month), the mice were euthanized using diethyl ether and blood samples were obtained. The serum samples were separated in order to measure the iron content, serum aspartate aminotransferase (AST), alanine aminotransferase (ALT) and alkaline phosphatase (ALP) levels. Livers were removed and kept in $10 \%$ buffered formalin for histopathological study. ${ }^{1}$

\section{Determination of total iron in serum}

The iron content was evaluated by microwave plasma-atomic emission spectroscopy (Agilent Technologies, USA). Iron standard solutions for calibration were obtained from singleelement stock solutions (Merck, Darmstadt, Germany) in 0.2\% $(\mathrm{w} / \mathrm{v})$ nitric acid. The mouse serum samples were examined for their iron content directly after 1 : 50 dilution with ultrapure water. ${ }^{1}$ Iron was expressed as $\mu \mathrm{g} \mathrm{dl}^{-1}$.

\section{Determination of serum ferric ion $\left(\mathrm{Fe}^{3+}\right)$}

$\mathrm{Fe}^{3+}$ concentration in serum was measured by an iron measuring kit (ZiestChem Diagnostics, Tehran, Iran). The iron attached to transferrin in mice serum samples in the acidic environment is usually released to form $\mathrm{Fe}^{3+}$ and is converted to $\mathrm{Fe}^{2+}$ through reducing agents. This ion creates a purple colored complex with Ferene. The color intensity is proportional to the amount of iron existing in the sample that can be evaluated at a wavelength of $600 \mathrm{~nm}$. Ferric content was expressed as $\mu \mathrm{g}$ $\mathrm{dl}^{-1}$.

\section{Determination of serum AST, ALT and ALP levels}

AST, ALT and ALP levels in separated serum samples were assessed with an autoanalyzer BT3000 PLUS (Biotechnica, Rome, Italy) based on the method of commercial kits (Pars Azmon Co., Tehran, Iran). Pars Azmon's AST Colorimetric Assay Kit is a rapid and simple assay in which AST catalyzes the transfer of an amino group from L-aspartate to 2-oxoglutatare and the products of this reversible transamination reaction are oxoglutarate and L-glutamate. The oxoglutatare and NADH are converted to malate and NAD by the enzyme malate dehydrogenase. The reduction in NADH absorbance at $340 \mathrm{~nm}$ is proportionate to AST activity. In Pars Azmon's ALT Colorimetric Assay Kit, an amino group is transferred from L-alanine to 2oxoglutatare. The products of this reversible transamination reaction include pyruvate and L-glutamate. ALT activity is measured by monitoring the rate of $\mathrm{NADH}$ oxidation in a coupled reaction system applying lactate dehydrogenase. The oxidation of $\mathrm{NADH}$ to $\mathrm{NAD}^{+}$is accompanied by a reduction in absorbance at $340 \mathrm{~nm}$. Under the conditions in which the ALT activity is rate limiting, the rate decrease is directly consistent with the ALT activity in the sample. The ALP Assay Kit is a very sensitive, simple and direct colorimetric assay designed to evaluate alkaline phosphatase (ALP) activity in serum and biological samples. The kit uses $p$-nitrophenyl phosphate as a phosphatase substrate which turns yellow $\left(\lambda_{\max }=405 \mathrm{~nm}\right)$ when it is dephosphorylated by ALP.

\section{Histology}

Formalin-fixed liver specimens for histology were put in paraffin wax. Tissue sections were cut at $5 \mathrm{~mm}$ and stained using H\&E and Prussian blue stain. The stained tissue sections were assessed under a light microscope (Lx 400, Labomed, California, USA).

\section{Statistical analysis}

Statistical analysis of the results was performed using IBM SPSS Statistics 21 and Excel 2013 for Windows. The results are expressed as means \pm standard deviation (SD). One way analysis of variance (ANOVA) was also carried out. The Tukey multiple comparison test was employed to identify the differences in means. All $p$ values lower than 0.05 were considered as significant.

\section{Conflicts of interest}

The authors declare that there is no conflict of interest.

\section{Acknowledgements}

This study was financially supported by a grant (no. 1250) from the Pharmaceutical Sciences Research Center, Mazandaran University of Medical Sciences, which we gratefully acknowledge.

\section{References}

1 M. Khalili, M. A. Ebrahimzadeh, M. Kosaryan, A. Abbasi and M. Azadbakht, RSC Adv., 2015, 5, 4804.

2 M. Savasari, M. Emadi, M. A. Bahmanyar and P. Biparva, J. Ind. Eng. Chem., 2015, 21, 1403.

3 M. Stefaniuk, P. Oleszczuk and Y. S. Ok, Chem. Eng. J., 2016, 287, 618.

4 A. Dias, A. Hussain, A. Marcos and A. Roque, Biotechnol. Adv., 2011, 29, 142.

5 C. W. Lim and I. S. Lee, Nano Today, 2010, 5, 412.

6 M. N. Nadagouda, A. B. Castle, R. C. Murdock, S. M. Hussain and R. S. Varma, Green Chem., 2010, 12, 114.

7 M. Bonder, Y. Zhang, K. Kiick, V. Papaefthymiou and G. Hadjipanayis, J. Magn. Magn. Mater., 2007, 311, 658.

8 T. Wang, J. Lin, Z. Chen, M. Megharaj and R. Naidu, J. Cleaner Prod., 2014, 83, 413. 
9 M. Pattanayak and P. Nayak, Int. J. Plant, Anim. Environ. Sci., 2013, 3, 68.

10 Z. Markova, P. Novak, J. Kaslik, P. Plachtova, M. Brazdova, D. Jancula, K. M. Siskova, L. Machala, B. Marsalek and R. Zboril, ACS Sustainable Chem. Eng., 2014, 2, 1674.

11 G. E. Hoag, J. B. Collins, J. L. Holcomb, J. R. Hoag, M. N. Nadagouda and R. S. Varma, J. Mater. Chem., 2009, 19, 8671.

12 W. A. Wannes, B. Mhamdi, J. Sriti, M. B. Jemia, O. Ouchikh, G. Hamdaoui, M. E. Kchouk and B. Marzouk, Food Chem. Toxicol., 2010, 48, 1362.

13 G. Alipour, S. Dashti and H. Hosseinzadeh, Phytother. Res., 2014, 28, 1125.

14 Z. Yi, X. Li, X. Xu, B. Luo, J. Luo, W. Wu, Y. Yi and Y. Tang, Colloids Surf., A, 2011, 392, 131.

15 M. Yoshimura, Y. Amakura, M. Tokuhara and T. Yoshida, J. Nat. Med., 2008, 62, 366.

16 G. K. Lopes, H. M. Schulman and M. Hermes-Lima, Biochim. Biophys. Acta, Gen. Subj., 1999, 1472, 142.

17 K. M. Kumar, B. K. Mandal, K. S. Kumar, P. S. Reddy and B. Sreedhar, Spectrochim. Acta, Part A, 2013, 102, 128.

18 Y. Fu, L. Liu, H.-Z. Yu, Y.-M. Wang and Q.-X. Guo, J. Am. Chem. Soc., 2005, 127, 7227.

19 S. Barua, R. Konwarh, S. S. Bhattacharya, P. Das, K. S. P. Devi, T. K. Maiti, M. Mandal and N. Karak, Colloids Surf., B, 2013, 105, 37.

20 G. Ghodake, S. R. Lim and D. S. Lee, Colloids Surf., B, 2013, 108, 147.

21 N. J. Reddy, D. N. Vali, M. Rani and S. S. Rani, Mater. Sci. Eng., C, 2014, 34, 115.

22 K. Ghasemi, Y. Ghasemi, A. Ehteshamnia, S. M. Nabavi, S. F. Nabavi, M. A. Ebrahimzadeh and F. Pourmorad, J. Med. Plants Res., 2011, 5, 1128.

23 A. Sousa, I. Ferreira, L. Barros, A. Bento and J. Pereira, LWTFood Sci. Technol., 2008, 41, 739.

24 M. Khalili, M. A. Ebrahimzadeh and M. Kosaryan, Int. J. Med. Mushrooms, 2015, 17, 847.

25 M. A. Ebrahimzadeh, S. M. Nabavi, S. F. Nabavi, B. Eslami and S. Ehsanifar, Pharmacologyonline, 2009, 2, 644.

26 M. A. Ebrahimzadeh, S. F. Nabavi, S. M. Nabavi and B. Eslami, Cent. Eur. J. Biol., 2010, 5, 338.
27 C. C. Chang, M. H. Yang, H. M. Wen and J. C. Chern, J. Food Drug Anal., 2002, 10, 178.

28 R. Willis, Analyst, 1998, 123, 435.

29 L. J. Porter, L. N. Hrstich and B. G. Chan, Phytochemistry, 1985, 25, 223.

30 H. Jiang, K. S. Moon, Z. Zhang, S. Pothukuchi and C. Wong, J. Nanopart. Res., 2006, 8, 117.

31 S. Kundu and U. Nithiyanantham, RSC Adv., 2013, 3, 25278.

32 K. K. H. Anand and B. K. Mandal, Spectrochim. Acta, Part A, 2015, 135, 639.

33 M. Arshad, A. Beg and Z. Siddiqui, Macromol. Mater. Eng., 1969, 7, 67.

34 D. Nayak, S. Ashe, P. R. Rauta, M. Kumari and B. Nayak, Mater. Sci. Eng., C, 2016, 58, 44.

35 P. J. Chen, S.-W. Tan and W. L. Wu, Environ. Sci. Technol., 2012, 46, 8431.

36 J. D. Clogston and A. K. Patri, Characterization of nanoparticles intended for drug delivery, 2011, p. 63.

37 C. M. Goodman, C. D. McCusker, T. Yilmaz and V. M. Rotello, Bioconjugate Chem., 2004, 15, 897.

38 W. A. Wannes and B. Marzouk, J. Food Drug Anal., 2016, 24, 316.

39 A. K. Mittal, A. Kaler and U. C. Banerjee, Nano Biomed. Eng., 2012, 4, 118.

40 A. Yıldırım, A. Mavi and A. A. Kara, J. Agric. Food Chem., 2001, 49, 4083.

41 C. G. Hadjipanayis, M. J. Bonder, S. Balakrishnan, X. Wang, H. Mao and G. C. Hadjipanayis, Small, 2008, 4, 1925.

42 S. A. Santos, J. J. Villaverde, C. S. Freire, M. R. M. Domingues, C. P. Neto and A. J. Silvestre, Ind. Crops Prod., 2012, 39, 120.

43 K. M. Kumar, B. K. Mandal and S. K. Tammina, RSC Adv., 2013, 3, 4033.

44 I. Bertini, H. B. Gray, S. J. Lippard and J. S. Valentine, Bioinorganic chemistry, University Science Books, 1994.

45 H. Najafzadeh, M. R. Jalali, H. Morovvati and F. Taravati, J. Med. Toxicol., 2010, 6, 22.

46 Y. Gao, N. Wang, Y. Zhang, Z. Ma, P. Guan, J. Ma, Y. Zhang, X. Zhang, J. Wang and J. Zhang, J. Ethnopharmacol., 2013, 145, 254. 